\title{
Bound on the Minimum Eigenvalue of $H$-Matrices Involving Hadamard Products
}

\author{
Kun Du, ${ }^{1}$ Guiding Gu, ${ }^{2}$ and Guo Liu ${ }^{1}$ \\ ${ }^{1}$ School of Finance, Shanghai University of Finance and Economics, Shanghai 200433, China \\ ${ }^{2}$ Department of Applied Mathematics, Shanghai University of Finance and Economics, Shanghai 200433, China
}

Correspondence should be addressed to Kun Du; d31415927@126.com

Received 5 March 2013; Accepted 4 June 2013

Academic Editor: Sorin Dascalescu

Copyright (C) 2013 Kun Du et al. This is an open access article distributed under the Creative Commons Attribution License, which permits unrestricted use, distribution, and reproduction in any medium, provided the original work is properly cited.

We present a new lower bound on the minimum eigenvalue of $H$-matrices involving Hadamard products $\tau\left(A_{1}^{\left(\alpha_{1}\right)} \circ \ldots \circ A_{m}^{\left(\alpha_{m}\right)}\right)$, and we show that our lower bound is larger than the lower bound $\prod_{k=1}^{m}\left[\tau\left(A_{k}\right)\right]^{\alpha_{k}}$. Three examples verify our result.

\section{Introduction}

In [1], it is shown by Theorem 5.7.15 that if $A_{k}$ are $n \times n H$ matrices for all $k \in\{1, \ldots, m\}$, and $\alpha_{k} \geq 0$ satisfy $\sum_{k=1}^{m} \alpha_{k} \geq 1$, then

$$
\tau\left(A_{1}^{\left(\alpha_{1}\right)} \circ \cdots \circ A_{m}^{\left(\alpha_{m}\right)}\right) \geq \prod_{k=1}^{m}\left[\tau\left(A_{k}\right)\right]^{\alpha_{k}},
$$

where $A^{(\alpha)}$ is defined as entrywise and any scalar definition of $a^{\alpha}$ such that $\left|a^{\alpha}\right|=|a|^{\alpha}$ is allowed. This theorem provided a beautiful result about the minimum eigenvalue of $H$-matrices involving Hadamard products, but sometimes this inequality could be very weak. For example,

$$
\begin{gathered}
A=\left(\begin{array}{ccc}
100 & -21 & -30 \\
0 & 1 & -12 \\
0 & 0 & 1
\end{array}\right), \quad B=\left(\begin{array}{ccc}
1 & 0 & 0 \\
-19 & 100 & 0 \\
-81 & -17 & 1
\end{array}\right), \\
C=\left(\begin{array}{ccc}
1 & 0 & 0 \\
0 & 1 & 0 \\
0 & 0 & 100
\end{array}\right)
\end{gathered}
$$

are $H$-matrices, and

$$
\tau\left(A^{(2)} \circ B^{(2)} \circ C^{(2)}\right)=10000 \gg \tau(A)^{2} \tau(B)^{2} \tau(C)^{2}=1,
$$

see the details in Section 3.

A lot of works have been done on the minimum eigenvalue of $M$-matrices and $H$-matrices involving Hadamard products, see the results in [1-6].
In this paper, we present a new lower bound by including diagonal entries and prove that our bound is larger than the bound in (1).

We now introduce some notations, see [1]. The Hadamard product of $A=\left[a_{i j}\right]_{m \times n}$ and $B=\left[b_{i j}\right]_{m \times n}$ is defined by $A \circ B \equiv$ $\left[a_{i j} b_{i j}\right]_{m \times n}$. We define $\left|a^{\alpha}\right|=|a|^{\alpha}$ and $0^{0}=1$. Let $A=\left[a_{i j}\right]_{n \times n}$ and denote $a_{i j}$ by $A(i, j)$ for every $i, j \in\{1, \ldots, n\}$. We denote $A^{(r)}=\left[a_{i j}^{r}\right]$, where $r \geq 0$, and denote by $Z_{n}$ the class of all $n \times n$ real matrices all of whose off-diagonal entries are nonpositive. Let $A \in Z_{n}$, then the minimum eigenvalue of $A$ is defined by $\tau(A)=\min \{\operatorname{Re}(\lambda): \lambda \in \sigma(A)\} ; \tau(A)$ is a eigenvalue of $A$.

For two real matrices $A, B \in Z_{n}$, the Fan product of $A$ and $B$, denoted by $A \star B=C=\left[c_{i j}\right]$, is defined by

$$
c_{i j}= \begin{cases}-a_{i j} b_{i j}, & \text { if } i \neq j, \\ a_{i i} b_{i i}, & \text { if } i=j,\end{cases}
$$

and then $A \star B \in Z_{n}$. The comparison matrix $M(A)=\left[m_{i j}\right]$ of a given matrix $A=\left[a_{i j}\right] \in \mathbb{C}^{n \times n}$ is defined by

$$
m_{i j}= \begin{cases}-\left|a_{i j}\right|, & \text { if } i \neq j, \\ \left|a_{i i}\right|, & \text { if } i=j .\end{cases}
$$

A matrix $A$ is an $H$-matrix if its comparison matrix $M(A)$ is an $M$-matrix. For $A \in \mathbb{C}^{n \times n}$, we define $\tau(A) \equiv \tau(M(A))$. 
Last, for $A \in Z_{n}$, we introduce a new definition $A^{[r]}=$ $\left[d_{i j}\right]$, where

$$
d_{i j}= \begin{cases}-\left|a_{i j}\right|^{r}, & \text { if } i \neq j, \\ \left|a_{i i}\right|^{r}, & \text { if } i=j\end{cases}
$$

\section{Main Results}

To prove the main theorem, we need several lemmas.

Lemma 1. Let $x_{j}=\left(x_{j}(1), \ldots, x_{j}(n)\right)^{T} \geq 0$ for all $j \in\{1, \ldots$, $m\}$. If $p_{j}>0$ and $\sum_{j=1}^{m}\left(1 / p_{j}\right)=r \geq 1$, then

$$
\sum_{i=1}^{n} \prod_{j=1}^{m} x_{j}(i) \leq \prod_{j=1}^{m}\left\{\sum_{i=1}^{n}\left[x_{j}(i)\right]^{p_{j}}\right\}^{1 / p_{j}} .
$$

Proof. When $r=1$, we can prove this inequality by the Hölder inequality and by induction. When $r>1$, apparently, $\sum_{j=1}^{m}\left(1 / r p_{j}\right)=1$, similarly, we can prove this inequality.

If $A \in Z_{n}$, then $A=\alpha I-B$ for some $B \geq 0$ and some $\alpha \in$ $\mathbb{R}$. Let $\sigma(B)=\left\{\rho(B), \lambda_{2}, \ldots, \lambda_{n}\right\}$. Then $\sigma(A)=\{\alpha-\rho(B), \alpha-$ $\left.\lambda_{2}, \ldots, \alpha-\lambda_{n}\right\}$. If there is a $\lambda_{i}$ such that $\alpha-\rho(B)=\operatorname{Re}(\alpha-$ $\rho(B))>\operatorname{Re}\left(\alpha-\lambda_{i}\right)=\alpha-\operatorname{Re}\left(\lambda_{i}\right)$, then $\rho(B)<\operatorname{Re}\left(\lambda_{i}\right)$, but this is a contradiction. So, $\tau(A)=\alpha-\rho(B)$. If $x \geq 0$ is the right Perron eigenvector of $B$, then $A x=(\alpha I-B) x=\alpha x-B x=$ $\alpha x-\rho(B) x=(\alpha-\rho(B)) x=\tau(A) x$. If $y \geq 0$ is the left Perron eigenvector of $B$, then $y^{T} A=y^{T}(\alpha I-B)=\alpha y^{T}-y^{T} B=$ $\alpha y^{T}-\rho(B) y^{T}=(\alpha-\rho(B)) y^{T}=\tau(A) y^{T}$. So, similar to the Perron-Frobenius theorem, we have the following: if $A \in Z_{n}$ became irreducible, then there exist positive vectors $u$ and $v$ such that $A u=\tau(A) u$, and $v^{T} A=\tau(A) v^{T}, u$ and $v$ being called right and left Perron eigenvectors of $A$, respectively.

Lemma 2. If $A \in Z_{n}$ is irreducible, and $A z \geq k z$ for a nonnegative nonzero vector $z$, then $k \leq \tau(A)$.

Proof. $A=\alpha I-P$, where $P \geq 0$ is irreducible and $\alpha \in \mathbb{R}$. By the Perron-Frobenius theorem, $P$ has a positive left Perron vector $y$, that is, $y^{T} P=\rho(P) y^{T}$. Note that $A z-k z=(\alpha-$ $k) z-P z \geq 0$. Hence, $y^{T}(A z-k z)=(\alpha-k) y^{T} z-\rho(P) y^{T} z=$ $(\alpha-k-\rho(P)) y^{T} z \geq 0$. Since $y^{T} z>0$, we have $k \leq \alpha-\rho(P)=$ $\tau(A)$.

Lemma 3. Let $A_{k} \in Z_{n}$ for all $k \in\{1, \ldots, m\}$. If $p_{k}>0$ and $\sum_{k=1}^{m}\left(1 / p_{k}\right) \geq 1$, then

$$
\begin{aligned}
& \tau\left(A_{1} \star \cdots \star A_{m}\right) \\
& \geq \min _{1 \leq i \leq n}\left\{\prod_{k=1}^{m} A_{k}(i, i)\right. \\
& \left.\quad-\prod_{k=1}^{m}\left[\left|A_{k}(i, i)\right|^{p_{k}}-\tau\left(A_{k}^{\left[p_{k}\right]}\right)\right]^{1 / p_{k}}\right\} .
\end{aligned}
$$

Proof. It is quite evident that the conclusion holds with equality for $n=1$.

For $n \geq 2$, we have two cases.

Case 1. $A_{1} \star \cdots \star A_{m} \in Z_{n}$ is irreducible. Then $A_{k} \in Z_{n}$ are irreducible for all $k \in\{1, \ldots, m\}$. Thus, $A_{k}^{\left[p_{k}\right]} \in Z_{n}$ are irreducible for all $k \in\{1, \ldots, m\}$. Let $u_{k}^{\left(p_{k}\right)}=\left(u_{k}(1)^{p_{k}}, \ldots\right.$, $\left.u_{k}(n)^{p_{k}}\right)^{T}>0$ be the right Perron eigenvectors of $A_{k}^{\left[p_{k}\right]}$ for all $k \in\{1, \ldots, m\}$. If $u_{k}^{(1)}=u_{k}=\left(u_{k}(1), \ldots, u_{k}(n)\right)^{T}>0$ for all $k \in\{1, \ldots, m\}$, then for every $i \in\{1, \ldots, n\}$, we have

$$
\begin{gathered}
A_{k}^{\left[p_{k}\right]} u_{k}^{\left(p_{k}\right)}=\tau\left(A_{k}^{\left[p_{k}\right]}\right) u_{k}^{\left(p_{k}\right)}, \\
\left|A_{k}(i, i)\right|^{p_{k}} u_{k}(i)^{p_{k}}-\sum_{j \neq i}\left|A_{k}(i, j)\right|^{p_{k}} u_{k}(j)^{p_{k}} \\
=\tau\left(A_{k}^{\left[p_{k}\right]}\right) u_{k}(i)^{p_{k}}, \\
\sum_{j \neq i}\left|A_{k}(i, j)\right|^{p_{k}} u_{k}(j)^{p_{k}} \\
=\left[\left|A_{k}(i, i)\right|^{p_{k}}-\tau\left(A_{k}^{\left[p_{k}\right]}\right)\right] u_{k}(i)^{p_{k}} \geq 0 .
\end{gathered}
$$

Let $C=A_{1} \star \cdots \star A_{m} \in Z_{n}$. Let $z=u_{1} \circ \cdots \circ u_{m}=$ $(z(1), \ldots, z(n))^{T}>0$. Then $z(i)=\prod_{k=1}^{m} u_{k}(i)$ for all $i \in$ $\{1, \ldots, n\}$. Then, for all $i \in\{1, \ldots, n\}$, we have

$$
\begin{aligned}
(C z)_{i}= & \prod_{k=1}^{m} A_{k}(i, i) z(i)-\sum_{j \neq i}\left(\prod_{k=1}^{m}\left|A_{k}(i, j)\right|\right) z(j) \\
= & \prod_{k=1}^{m} A_{k}(i, i) z(i)-\sum_{j \neq i} \prod_{k=1}^{m}\left(\left|A_{k}(i, j)\right| u_{k}(j)\right) \\
\geq & \prod_{k=1}^{m} A_{k}(i, i) z(i) \\
& \left.-\prod_{k=1}^{m}\left\{\sum_{j \neq i}\left|A_{k}(i, j)\right|^{p_{k}} u_{k}(j)^{p_{k}}\right\}\right]^{1 / p_{k}} \\
= & \prod_{k=1}^{m} A_{k}(i, i) z(i) \\
& -\prod_{k=1}^{m}\left\{\left[\left|A_{k}(i, i)\right|^{p_{k}}-\tau\left(A_{k}^{\left[p_{k}\right]}\right)\right] u_{k}(i)^{p_{k}}\right\}^{1 / p_{k}} \\
= & \left\{\prod_{k=1}^{m} A_{k}(i, i)\right. \\
& \left.-\prod_{k=1}^{m}\left[\left|A_{k}(i, i)\right|^{p_{k}}-\tau\left(A_{k}^{\left[p_{k}\right]}\right)\right]^{1 / p_{k}}\right\}(i) . \\
&
\end{aligned}
$$


By Lemma 1, the "z" hold. By Lemma 2, we have

$$
\begin{aligned}
& \tau\left(A_{1} \star \cdots \star A_{m}\right) \\
& \geq \min _{1 \leq i \leq n}\left\{\prod_{k=1}^{m} A_{k}(i, i)\right. \\
&\left.\quad-\prod_{k=1}^{m}\left[\left|A_{k}(i, i)\right|^{p_{k}}-\tau\left(A_{k}^{\left[p_{k}\right]}\right)\right]^{1 / p_{k}}\right\} .
\end{aligned}
$$

Case 2. $A_{1} \star \cdots \star A_{m} \in Z_{n}$ is reducible. We denote by $T$ the $n \times n$ permutation matrix $\left(t_{i j}\right)$ with

$$
t_{12}=t_{23}=\cdots=t_{n-1, n}=t_{n 1}=1,
$$

the remaining $t_{i j}$ zero, then both $A_{k}-\varepsilon T \in Z_{n}$ are irreducible for any chosen positive real number $\varepsilon$. Now we substitute $A_{k}-$ $\varepsilon T$ for $A_{k}$ in the previous case, and then by letting $\varepsilon \rightarrow 0$, the result follows by continuity.

Lemma 4 (see [7]). Let for all $i \in\{1, \ldots, n\}, x_{i} \geq y_{i} \geq 0$ and $p_{i}>0$ satisfying $\sum_{i=1}^{n}\left(1 / p_{i}\right) \geq 1$. Then

$$
\prod_{i=1}^{n} x_{i}-\prod_{i=1}^{n} y_{i} \geq \prod_{i=1}^{n}\left(x_{i}^{p_{i}}-y_{i}^{p_{i}}\right)^{1 / p_{i}}
$$

The following is our main theorem.

Theorem 5. Let $\alpha_{k} \geq 0$ and $\sum_{k=1}^{m} \alpha_{k} \geq 1$. If $A_{k}$ is $n \times n \mathrm{H}$ matrices for all $k \in\{1, \ldots, m\}$, then

$$
\begin{gathered}
\tau\left(A_{1}^{\left(\alpha_{1}\right)} \circ \ldots \circ A_{m}^{\left(\alpha_{m}\right)}\right) \\
\geq \min _{1 \leq i \leq n}\left\{\prod_{k=1}^{m}\left|A_{k}(i, i)\right|^{\alpha_{k}}\right. \\
\left.\quad-\prod_{k=1}^{m}\left[\left|A_{k}(i, i)\right|-\tau\left(A_{k}\right)\right]^{\alpha_{k}}\right\} \\
\geq \prod_{k=1}^{m}\left[\tau\left(A_{k}\right)\right]^{\alpha_{k}} .
\end{gathered}
$$

Proof. Without loss of generality, we can assume that $\alpha_{k}>0$ for all $k \in\{1,2, \ldots, m\}$. Let $p_{k}=1 / \alpha_{k}>0$, then $\sum_{k=1}^{m}\left(1 / p_{k}\right)=$ $\sum_{k=1}^{m} \alpha_{k} \geq 1$. Now we have:

$$
\begin{aligned}
\tau & \left(A_{1}^{\left(\alpha_{1}\right)} \circ \cdots \circ A_{m}^{\left(\alpha_{m}\right)}\right) \\
& =\tau\left(M\left(A_{1}^{\left(\alpha_{1}\right)} \circ \cdots \circ A_{m}^{\left(\alpha_{m}\right)}\right)\right) \\
& =\tau\left(M\left(A_{1}^{\left(\alpha_{1}\right)}\right) \star \cdots \star M\left(A_{m}^{\left(\alpha_{m}\right)}\right)\right)
\end{aligned}
$$

$$
\begin{aligned}
& \geq \min _{1 \leq i \leq n}\left\{\prod_{k=1}^{m}\left|A_{k}(i, i)\right|^{\alpha_{k}}\right. \\
& \left.\quad-\prod_{k=1}^{m}\left[\left|A_{k}(i, i)\right|-\tau\left(M\left(A_{k}^{\left(\alpha_{k}\right)}\right)^{\left[p_{k}\right]}\right)\right]^{1 / p_{k}}\right\} \\
& =\min _{1 \leq i \leq n}\left\{\prod_{k=1}^{m}\left|A_{k}(i, i)\right|^{\alpha_{k}}\right. \\
& \left.\quad-\prod_{k=1}^{m}\left[\left|A_{k}(i, i)\right|-\tau\left(A_{k}\right)\right]^{\alpha_{k}}\right\} \\
& =\min _{1 \leq i \leq n} \prod_{k=1}^{m}\left[\left|A_{k}(i, i)\right|-\left(\left|A_{k}(i, i)\right|-\tau\left(A_{k}\right)\right)\right]^{1 / p_{k}} \\
& =\prod_{k=1}^{m}\left[\tau\left(A_{k}\right)\right]^{\alpha_{k}} .
\end{aligned}
$$

The first " $\geq$ " hold by Lemma 3 . Because $A_{k}$ is $H$-matrix, we have $0 \leq \tau\left(A_{k}\right) \leq\left|A_{k}(i, i)\right|$, which means that $\left|A_{k}(i, i)\right| \geq$ $\left|A_{k}(i, i)\right|-\tau\left(A_{k}\right) \geq 0$. So the second " $\geq$ " hold by $\left|A_{k}(i, i)\right|^{\alpha_{k}} \geq$ $\left[\left|A_{k}(i, i)\right|-\tau\left(A_{k}\right)\right]^{\alpha_{k}} \geq 0$ and Lemma 4.

\section{Examples}

In this section, we present three examples to illustrate our improved bound.

Example 6. We take the matrices $A, B$, and $C$ in Section 1. It is easy to get $\tau(A)=\tau(B)=\tau(C)=1$, so the low bound in (1) is $\tau(A)^{2} \tau(B)^{2} \tau(C)^{2}=1$; on the other side, we can get $\tau\left(A^{(2)} \circ B^{(2)} \circ C^{(2)}\right)=10000$, which is greatly larger than the lower bound $\tau(A)=\tau(B)=\tau(C)$, that is,

$$
\tau\left(A^{(2)} \circ B^{(2)} \circ C^{(2)}\right)=10000 \gg \tau(A)^{2} \tau(B)^{2} \tau(C)^{2}=1 .
$$

However, our lower bound in Theorem 5 is

$$
\begin{aligned}
\min _{1 \leq i \leq 3}\left\{a_{i i}^{2} b_{i i}^{2} c_{i i}^{2}-\left[a_{i i}-\tau(A)\right]^{2}\right. \\
\left.\times\left[b_{i i}-\tau(B)\right]^{2}\left[c_{i i}-\tau(C)\right]^{2}\right\} \\
=\min _{1 \leq i \leq 3}\left\{100^{2} \times 1^{2} \times 1^{2}-(100-1)^{2}\right. \\
\left.\times(1-1)^{2}(1-1)^{2}\right\}=10000,
\end{aligned}
$$

which is the exact value of $\tau\left(A^{(2)} \circ B^{(2)} \circ C^{(2)}\right)$.

Example 7. Let

$$
A=\left(\begin{array}{ccc}
11 & -5 & -8 \\
-2 & 14 & -7 \\
-1 & -3 & 9
\end{array}\right), \quad B=\left(\begin{array}{ccc}
9 & -8 & -7 \\
-2 & 13 & -4 \\
-1 & -6 & 15
\end{array}\right) .
$$

It is easy to see that $A$ and $B$ are $H$-matrices and $\tau(A)=$ 3.9152, $\tau(B)=4.1345$. Thus, we have

$$
\tau\left(A^{(2)} \circ B^{(2)}\right)=9799>\tau(A)^{2} \tau(B)^{2}=262.0319,
$$


but our bound is

$$
\min _{1 \leq i \leq 3}\left\{a_{i i}^{2} b_{i i}^{2}-\left[a_{i i}-\tau(A)\right]^{2}\left[b_{i i}-\tau(B)\right]^{2}\right\}=8612.8,
$$

which is close to the exact value of 9799.

Example 8. Take the $H$-matrices

$$
A=\left(\begin{array}{ccc}
15 & -12 & -4 \\
-3 & 14 & -2 \\
-4 & 0 & 11
\end{array}\right), \quad B=\left(\begin{array}{ccc}
27 & -14 & -6 \\
-4 & 20 & -8 \\
-10 & -5 & 23
\end{array}\right)
$$

It is easy to see that

$$
\tau\left(A^{(2)} \circ B^{(1)}\right)=2775.5>\tau(A)^{2} \tau(B)^{1}=244.0,
$$

but our bound is

$$
\min _{1 \leq i \leq 3}\left\{a_{i i}^{2} b_{i i}^{1}-\left[a_{i i}-\tau(A)\right]^{2}\left[b_{i i}-\tau(B)\right]^{1}\right\}=2340.3 .
$$

Also, our bound is much closer to the exact value than the low bound in (1).

\section{Acknowledgments}

The authors would like to thank the anonymous reviewers for their helpful comments and valuable suggestions which improved the quality of this paper. This work is supported by the Cultivation Fund of the Key Scientific and Technical Innovation Project, Ministry of Education of China (no. 708040), the Leading Academic Discipline Program, 211 Project for Shanghai University of Finance and Economics (the third phase), and Graduate Research and Innovation Fund of Shanghai University of Finance and Economics (CXJJ-2012-385).

\section{References}

[1] R. A. Horn and C. R. Johnson, Topics in Matrix Analysis, Cambridge University Press, Cambridge, UK, 1991.

[2] H.-B. Li, T.-Z. Huang, S.-Q. Shen, and H. Li, "Lower bounds for the minimum eigenvalue of Hadamard product of an $M$-matrix and its inverse," Linear Algebra and Its Applications, vol. 420, no. 1, pp. 235-247, 2007.

[3] M. Fiedler and T. L. Markham, "An inequality for the Hadamard product of an $M$-matrix and an inverse $M$-matrix," Linear Algebra and Its Applications, vol. 101, pp. 1-8, 1988.

[4] M. Fang, "Bounds on eigenvalues of the Hadamard product and the Fan product of matrices," Linear Algebra and Its Applications, vol. 425, no. 1, pp. 7-15, 2007.

[5] P. N. Shivakumar, J. J. Williams, Q. Ye, and C. A. Marinov, "On two-sided bounds related to weakly diagonally dominant $M$ matrices with application to digital circuit dynamics," SIAM Journal on Matrix Analysis and Applications, vol. 17, no. 2, pp. 298-312, 1996.

[6] L. M. Zou, Y. Y. Jiang, J. K. Liu, and Y. M. Feng, "Bounds on the minimum eigenvalue of $M$-matrices," Southeast Asian Bulletin of Mathematics, vol. 34, no. 6, pp. 1165-1169, 2010.

[7] G. H. Hardy, J. E. Littlewood, and G. Polyn, Inequalities, Cambridge University Press, Cambridage, UK, 1934. 


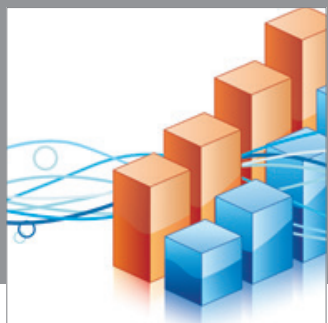

Advances in

Operations Research

mansans

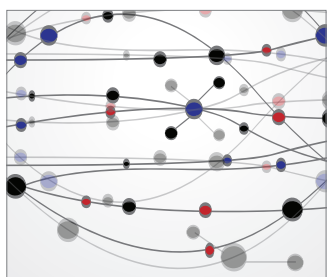

The Scientific World Journal
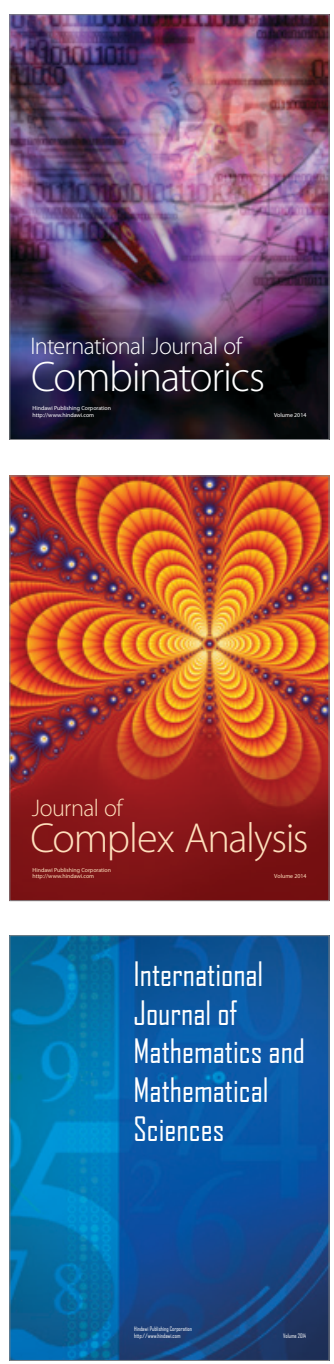
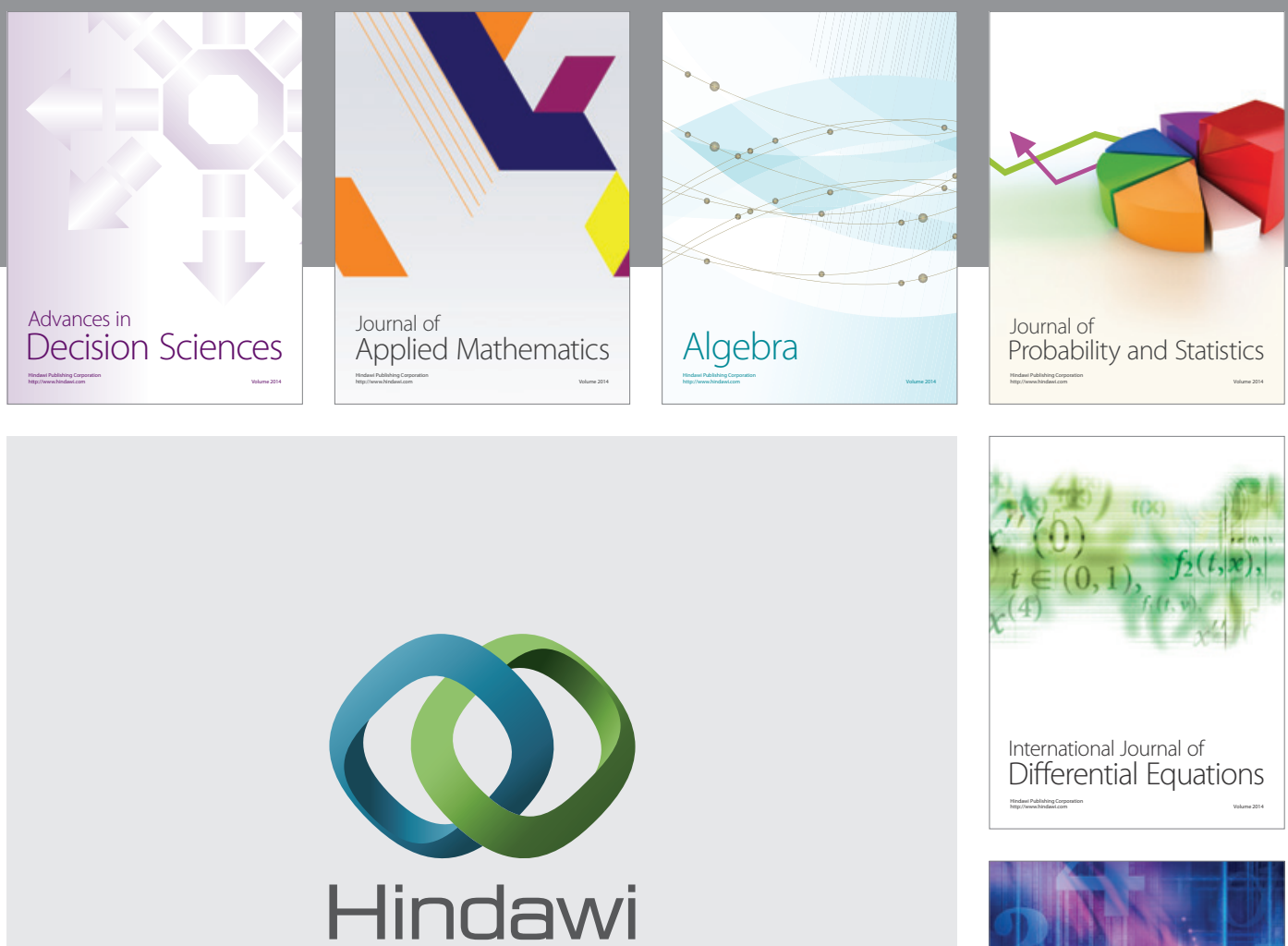

Submit your manuscripts at http://www.hindawi.com
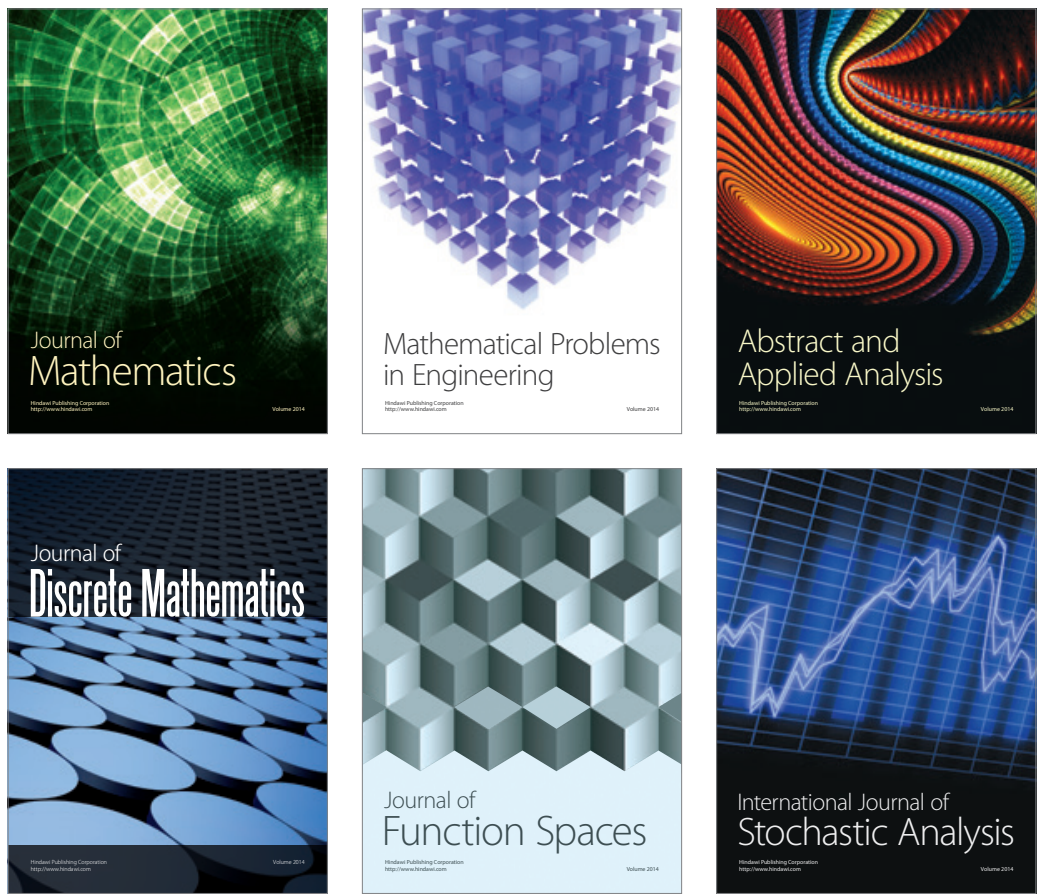

Journal of

Function Spaces

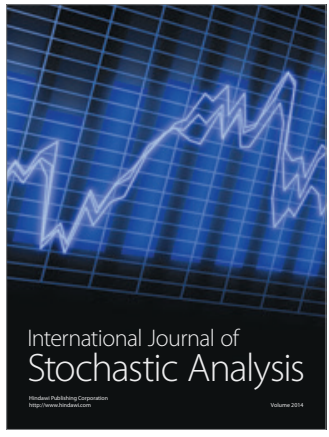

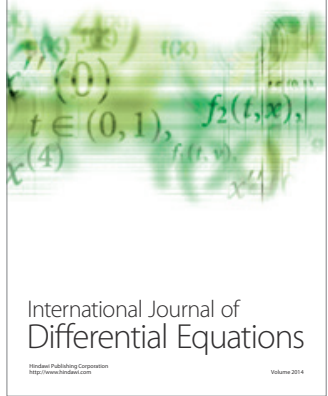
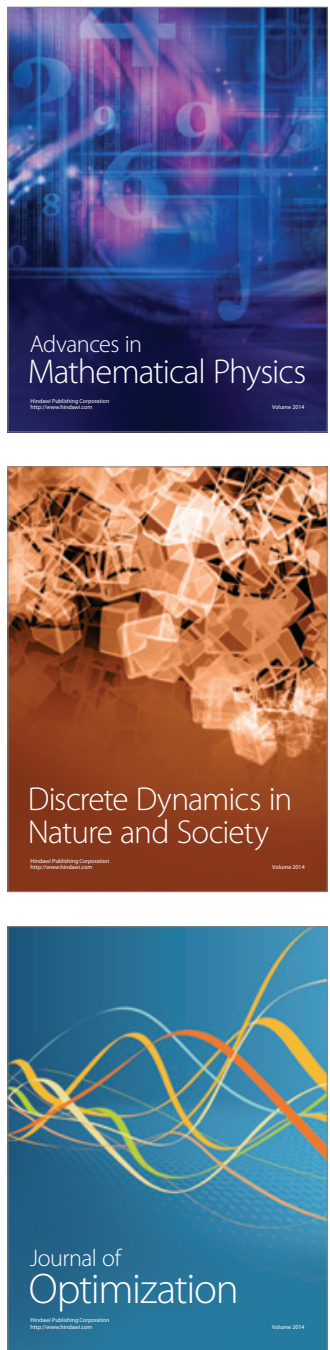